\title{
MODELOS DE RETENÇÃO DE ESTUDANTES: ABORDAGENS E PERSPECTIVAS ${ }^{1}$
}

\author{
Oberdan Santos da Costa ${ }^{2}$
}

Luis Borges Gouveia ${ }^{3}$

http://dx.doi.org/10.1590/1413-2311.226.85489

\section{RESUMO}

Diferentes abordagens e perspectivas têm sido utilizadas nos modelos teóricos de retenção de estudantes nas últimas oito décadas, com foco nas altas taxas da evasão de estudante no ensino superior. Muitas dessas abordagens e perspectivas parecem ser pouco conhecidas dos educadores, administradores e formuladores de políticas das instituições de ensino em função das suas complexidades. Alguns pesquisadores, preocupados com as questões de evasão escolar, estão saindo de uma abordagem com perspectiva pontual para uma abordagem mais abrangente e integrativa, preocupada com soluções mais construtivas. Esse estudo tem como objetivo identificar e compreender as perspectivas dos modelos teóricos de retenção e fornecer aos educadores, administradores e formuladores de políticas uma visão abrangente dos principais modelos teóricos de retenção de estudantes, considerando as abordagens e perspectivas para auxiliar nas questões relativas à evasão de estudante.A pesquisa resultou em vinte e três modelos teóricos de retenção com suas respectivas abordagens e perspectivas.

Palavras-chave: Retenção. Evasão. Estudantes. Abordagens. Perspectivas.

\footnotetext{
${ }^{1}$ Recebido em 02/08/2018, aprovado em 31/10/2018.

${ }^{2}$ Universidade Fernando Pessoa (UFP) - Faculdade de Ciência e Tecnologia; Porto (Portugal) sc.oberda@gmail.com

3 Universidade Fernando Pessoa (UFP) - Faculdade de Ciência e Tecnologia; Porto (Portugal) $\underline{\operatorname{lmbg} @ \text { ufp.edu.pt }}$
} 


\title{
THEORETICAL MODELS OF STUDENT RETENTION: APPROACHES AND PERSPECTIVES
}

\begin{abstract}
Different approaches and perspectives have been used in the theoretical models of student retention in the last eight decades focusing on high rates of student dropout in higher education. Many of these approaches and perspectives seem to be poorly understood by educators, administrators, and policy makers of educational institutions because of their complexities. Some researchers concerned with student withdrawal issues are moving from a one-on-one perspective to a more comprehensive and integrative approach, concerned with more constructive solutions. This study aims to identify and understand the perspectives of theoretical retention models and to provide educators, administrators and policymakers with a comprehensive view of the main theoretical models of student retention, considering approaches and perspectives to assist student evasion issues. The research resulted in twenty three theoretical retention models with their respective approaches and perspectives.
\end{abstract}

Keywords: Retention. Dropout. Students. Approaches. Perspectives.

\section{MODELOS TEÓRICOS DE RETENCIÓN DE ESTUDIANTES: ABORDAJES Y PERSPECTIVAS}

\section{RESUMEN}

Se han utilizado diferentes enfoques y perspectivas en los modelos teóricos de retención de estudiantes en las últimas ocho décadas, centrándose en las altas tasas de abandono escolar en la educación superior. Muchos de estos enfoques y perspectivas parecen ser poco comprendidos por los educadores, administradores y responsables de las políticas de las instituciones educativas debido a sus complejidades. Algunos investigadores preocupados por los problemas de retiro de estudiantes están pasando de una perspectiva individual a un enfoque más integral e integrador, preocupado por soluciones más constructivas. El objetivo de este estudio es identificar y comprender las perspectivas de los modelos de retención teóricos y proporcionar a los educadores, administradores y formuladores de políticas una 
visión integral de los principales modelos teóricos de retención de estudiantes, considerando enfoques y perspectivas para ayudar con los problemas de evasión de estudiantes. La investigación resultó en veintitrés modelos de retención teórica con sus respectivos enfoques y perspectivas.

Palabras clave: Retención. Abandono. Estudiantes. Enfoques. Perspectivas.

\section{INTRODUÇÃO}

Considerando a vasta literatura dos modelos teóricos sobre retenção nas últimas oito décadas, com foco nas altas taxas de não conclusão, essa evidencia o grande desafio das instituições de ensino superior (IES) de reter seus estudantes. Muitas questões relacionadas a retenção de estudantes têm sido abordadas por estudiosos e de diferentes perspectivas para proposição de soluções. Algumas dessas questões podem estar relacionadas a metodologia aplicadas aos conceitos e as definições do termo retenção pelos estudiosos, que em alguns casos é entendido, ou mesmo confundido, com o termo persistência. As várias questões relacionadas ao problema da evasão dos estudantes têm feito com que os pesquisadores saiam de uma abordagem com perspectiva pontual para uma abordagem mais abrangente e integrativa, preocupada com soluções mais construtivas. Este estudo tem como objetivo identificar e compreender as perspectivas dos modelos teóricos de retenção e fornecer aos educadores, aos administradores e aos formuladores de políticas uma visão abrangente dos principais modelos teóricos de retenção de estudantes, considerando as abordagens e perspectivas para auxiliar nas questões da evasão estudantil. Esta pesquisa é do tipo exploratória, pois o método envolve o uso de recursos de biblioteca on-line para o levantamento de trabalhos de teses, dissertações e artigos científicos na literatura nacional e internacional. As bases de dados pesquisadas incluíram o seguinte: Google Scholar e EBSCO Host. Os termos-chave que se encaixam nos domínios deste estudo foram: Modelo, retenção, abordagem, perspectiva e ensino superior.

Os primeiros estudos de retenção de graduação ocorreram nos Estados Unidos na década de 1930 e centraram-se no que foi referido na época como mortalidade estudantil. A teoria do suicídio (DURKHEIM, 1951) do campo da sociologia, as visões teóricas dos ritos de passagem nas sociedades tribais (VAN GENNEP, 1960) no campo da antropologia social e do conceito de roteamento do trabalho do campo dos recursos humanos (PRICE, 1977) foram teorias fundamentais para o estudo e desenvolvimento dos modelos de retenção. De 
acordo com Berger, Ram'rez e Lyons (2012), em 1970, a era da construção de teorias de retenção havia começado, em grande parte com o trabalho de Spady (1971), "Dropouts from Higher Education: An Interdisciplinary Review and Synthesis". Este foi o primeiro modelo sociológico de retenção de estudantes. De acordo com Spady (1971), existem dois sistemas em cada faculdade (acadêmica e social) e pelo menos dois fatores em cada sistema que influenciam a decisão do aluno de se retirar: no acadêmico, notas e desenvolvimento intelectual no sistema acadêmico, e, no sistema social, congruência normativa e apoio de amizade. Após o trabalho de Spady, estudos e modelos posteriores levaram em conta a natureza das relações institucionais dos alunos. Na década de 1970, a introdução do termo "retenção" para descrever a persistência dos alunos incluiu o conceito de que as instituições compartilhavam responsabilidade em influenciar as decisões dos alunos em relação ao "abandono" (HABLEY; BLOOM; ROBBINS, 2012). Desde então, muitos estudos de retenção de estudantes foram realizados e modelos teóricos foram desenvolvidos. A exemplo, se tem o modelo de integração do estudante de Tinto (1975), que alicerçou muitos estudos sobre o tema abandono e retenção. No Brasil, o estudo envolvendo retenção foi iniciado em 1995 e concluído em 1996, sendo realizado por uma Comissão Especial instituída pela Secretaria de Educação Superior do Ministério da Educação e do Desporto (SESu/MEC). O estudo reuniu um conjunto de dados relativos aos índices de diplomação, retenção e evasão dos cursos de graduação de cerca de $90 \%$ das instituições federais de ensino superior (IFES) brasileiras à época (BRASIL, 1997).

Este trabalho está estruturado da seguinte forma: dentro da segunda seção, a visão histórica da retenção de estudantes na graduação. A terceira seção, definição de retenção e termo relacionado. A próxima seção (quarta), principais modelos teóricos de retenção de estudantes, abordagem e perspectivas. $\mathrm{Na}$ quinta seção, tipos de retenção. Na sexta seção, fatores chaves relacionados à retenção de estudantes. Na sétima seção, indicadores de retenção. Na oitava seção, desenvolvimentos futuros e limitações das teorias da retenção de estudantes. A seção final, considerações finais.

\section{VISÃO HISTÓRICA DA RETENÇÃO DE ESTUDANTES NA GRADUAÇÃO}

Os desafios para retenção de estudantes na graduação nas instituições de ensino superior nos Estados Unidos da América teve início no ano 1930, período em que as instituições atendiam a um público selecionado, a conclusão do grau de estudante era rara e as universidades tinham um foco mais para a sobrevivência institucional do que na graduação 
estudantil, mas com o crescimento das cidades e mudanças nos estilos de vida das pessoas, as demandas por um diploma de graduação aumentaram (BERGER; RAM'REZ; LYON, 2005). Diante de novo cenário de mudanças, o interesse pelos estudos de retenção na graduação começou a crescer. Assim, os autores informam que os modelos teóricos decorrentes do estudo sistemático do fenômeno não foram desenvolvidos até ao início da década de 1970. Os autores dividiram o desenvolvimento de estudos de retenção de estudantes cronologicamente em nove épocas:
a) Pré-história da Retenção no período de 1600 a 1800;
b) Evoluindo para a retenção no período entre 1800 a 1900;
c) Desenvolvimentos iniciais no período de 1900 a 1950;
d) Lidando com expansão no período que se inicia na década de 50;
e) Prevenção de abandono na década de 60;
f) Construindo teorias na década de 70;
g) Gerenciando inscrição na década de 80;
h) Avançando nos horizontes na década de 90;
i) Início do século XXI período de tendências atuais e futuras.

\section{DEFINIÇÃO DE RETENÇÃO E TERMO RELACIONADO}

Mesmo depois de mais de oito décadas de pesquisa sobre retenção, a definição de retenção de estudantes, ainda permanece ambígua, não havendo um consenso entre os estudiosos do assunto. Summerskill (1962) é talvez um dos primeiros a solicitar o desenvolvimento de padrões nacionais em relação à definição e mensuração da retenção, incluindo taxas de retorno e conclusão anual. Em uma investigação feita pela Universidade Nacional da Colombia (2002), é relatado nos estudos, que não há diferença entre os termos retenção e persistência. No entanto, uma revisão de literatura internacional sobre o assunto permitiu estabelecer que alguns autores consideram importante diferenciar esses dois termos. O Centro Nacional para Educação Estatística dos Estados Unidos corrobora com a segunda linha, e explica que a retenção institucional é definida como a porcentagem dos alunos que completam um programa, ou mantêm inscrição em sua primeira instituição, e, a persistência, é a porcentagem de estudantes que completam um programa ou mantem sua inscrição em qualquer nível de instituição pós-secundário (NATIONAL CENTER FOR EDUCATION STATISTICS, 2000). Alguns termos podem ter significados diferentes fora do contexto do estudo. No contexto da educação superior a retenção é percebida como um fenômeno positivo 
na medida em que os alunos permanecem na universidade para alcançar o objetivo desejado, mesmo diante de fatores complexos e multidimensionais (culturais, sociais, institucionais e individuais). A maioria das definições apresentadas são de autores internacionais, especialmente norte-americanos. O termo relacionado à retenção, conforme cada autor e a definição por estes empregadas estão descritos na coluna termo relacionado. Após a revisão das definições adotados pelos autores e termo relacionado ao processo de retenção, se estabelece uma definição mais adequada com base neste estudo. O Quadro 1 representa os autores que diferenciam e não diferenciam os termos retenção e persistência, assim como autores que definem somente retenção.

\section{Quadro 1 - Retenção (RTC) versus Persistência (PRT)}

\begin{tabular}{|c|c|c|c|}
\hline & & Autores que diferenciam o termo de retenção & \\
\hline & Autor & Definição & $\begin{array}{c}\text { Termo } \\
\text { relacionado }\end{array}$ \\
\hline & Astin (1975) & $\begin{array}{l}\text { RTC: É a capacidade da instituição para manter os alunos de um } \\
\text { ano para o próximo } \\
\text { PRT: É a capacidade ou motivação de um aluno para atingir } \\
\text { seus próprios objetivos acadêmicos }\end{array}$ & Abandono \\
\hline & Swail (1995) & $\begin{array}{l}\text { RTC: É um termo usado para descrever o processo de } \\
\text { atendimento contínuo dos alunos em uma instituição para obter } \\
\text { seu diploma } \\
\text { PRT: É a capacidade de um aluno ou grupo de estudantes } \\
\text { permanecerem em uma faculdade ou instituição }\end{array}$ & Abandono \\
\hline$\frac{\pi}{0}$ & $\begin{array}{l}\text { National Center } \\
\text { for Education } \\
\text { Statistics } \\
(2000)\end{array}$ & $\begin{array}{l}\text { RTC: Refere-se aos alunos que permanecem na mesma } \\
\text { instituição desde o momento em que começam até terminar um } \\
\text { programa } \\
\text { PRT: Refere-se a todos os alunos que continuam matriculados } \\
\text { até terminar um programa e, portanto, inclui aqueles que obtêm } \\
\text { seu diploma }\end{array}$ & - \\
\hline  & $\begin{array}{l}\text { Berger, } \\
\text { Ram'rez e } \\
\text { Lyon (2005) }\end{array}$ & $\begin{array}{l}\text { RTC: É capacidade institucional para manter um Aluno da } \\
\text { admissão / inscrição até a graduação. } \\
\text { PRT: Afirmam que a persistência é o desejo e a ação de um } \\
\text { aluno de permanecer dentro de um sistema de ensino superior } \\
\text { desde o início até obter seu título }\end{array}$ & Persistência \\
\hline
\end{tabular}




\begin{tabular}{|c|c|c|}
\hline $\begin{array}{l}\text { National Center } \\
\text { for Education } \\
\text { Statistics } \\
\text { (2012) }\end{array}$ & $\begin{array}{l}\text { RTC: É uma medida da taxa em que os estudantes persistem em } \\
\text { seu programa educacional em uma instituição, expressa em } \\
\text { percentagem. } \\
\text { PRT:refere a continuação do curso de um curso de semestre a } \\
\text { semestre que leva à graduação. }\end{array}$ & Persistência \\
\hline \multicolumn{3}{|c|}{ Autores que não diferenciam o termo de retenção } \\
\hline Autor & Definições & $\begin{array}{c}\text { Termo } \\
\text { relacionado }\end{array}$ \\
\hline $\begin{array}{l}\text { Universidade } \\
\text { da Califórnia } \\
\text { (1994 apud } \\
\text { GUEVARA, } \\
\text { 2010) }\end{array}$ & $\begin{array}{l}\text { RTC ou PRT: Afirma que a persistência e a retenção referem-se } \\
\text { ao ato de se manter matriculado em uma faculdade ou } \\
\text { universidade, enquanto o abandono e aposentadoria refere-se ao } \\
\text { ato de sair. }\end{array}$ & Persistência \\
\hline Himmel (2002) & $\begin{array}{l}\text { RTC ou PRT: Define a retenção como "a persistência dos alunos } \\
\text { em um programa de estudos universitários até atingir seu } \\
\text { diploma ou grau". }\end{array}$ & Persistência \\
\hline $\begin{array}{l}\text { Argentina } \\
(2003)\end{array}$ & $\begin{array}{l}\text { RTC ou PRT: A retenção escolar é entendida como "a } \\
\text { capacidade do sistema educacional para alcançar permanência } \\
\text { dos alunos nas salas de aula, garantindo a realização de ciclos e } \\
\text { níveis nos horários previstos e garantindo o domínio das } \\
\text { competências e conhecimentos correspondentes" }\end{array}$ & - \\
\hline \multicolumn{3}{|c|}{ Autores que definem somente a retenção } \\
\hline Autor & Definições & $\begin{array}{c}\text { Termo } \\
\text { relacionado }\end{array}$ \\
\hline $\begin{array}{l}\text { Lenning, Beal e } \\
\text { Sauer (1980) }\end{array}$ & $\begin{array}{l}\text { RTC: É aquela que ocorre quando os alunos completam, } \\
\text { continuam ou retomam seus estudos. }\end{array}$ & Desgaste \\
\hline Bean (1980) & $\begin{array}{l}\text { RTC: Afirma que o estudante que se integra com sucesso a uma } \\
\text { universidade ou faculdade, tem uma experiência bem-sucedida e } \\
\text { persiste. }\end{array}$ & $\begin{array}{l}\text { desgaste e } \\
\text { Sucesso }\end{array}$ \\
\hline Tinto (1993) & $\begin{array}{l}\text { RTC: Estudantes que conhecem objetivos educacionais } \\
\text { claramente definidos, sejam eles créditos de curso, progresso na } \\
\text { carreira ou realização de novas habilidades }\end{array}$ & Progresso \\
\hline Kerka (1995) & $\begin{array}{l}\text { RTC: É "manter os alunos nos programas até atingir seus } \\
\text { objetivos" }\end{array}$ & Sucesso \\
\hline
\end{tabular}




\begin{tabular}{|c|c|c|}
\hline Santos (1999) & $\begin{array}{l}\text { Condição do aluno regularmente matriculado no curso de } \\
\text { origem quando da realização do estudo. }\end{array}$ & - \\
\hline Levitz (2001) & $\begin{array}{l}\text { RTC: refere-se a Conclusão bem-sucedida dos objetivos } \\
\text { acadêmicos dos estudantes de obtenção de graus. }\end{array}$ & - \\
\hline $\begin{array}{l}\text { Hagedorn } \\
(2006)\end{array}$ & $\begin{array}{l}\text { RTC: é permanecer na faculdade ou universidade até obter um } \\
\text { título. }\end{array}$ & Persistência \\
\hline $\begin{array}{l}\text { Pascarella e } \\
\text { Terenzini } \\
(2005)\end{array}$ & $\begin{array}{l}\text { RTC: é a rematrícula progressiva na faculdade, de um período } \\
\text { para o próximo, se contínua, ou então retomada, se } \\
\text { temporariamente interrompida. }\end{array}$ & Persistência \\
\hline $\begin{array}{l}\text { Seidman } \\
(2005)\end{array}$ & $\begin{array}{l}\text { RTC: é a capacidade de uma faculdade ou universidade de } \\
\text { graduar com sucesso os alunos que inicialmente se inscreveram } \\
\text { na instituição }\end{array}$ & Sucesso \\
\hline $\begin{array}{l}\text { Glossário de } \\
\text { Ensino } \\
\text { Superior na } \\
\text { América Latina } \\
\text { e Caribe ( } 2007 \\
\text { apud } \\
\text { GUEVARA, } \\
\text { 2010) }\end{array}$ & $\begin{array}{l}\text { RTC: É a "capacidade de uma instituição ou carreira de ensino } \\
\text { superior para garantir que o número de alunos matriculados nele } \\
\text { abandone ou deserte." }\end{array}$ & Abandono \\
\hline $\begin{array}{l}\text { Universidad } \\
\text { Tecnológica de } \\
\text { Pereira (2008) }\end{array}$ & $\begin{array}{l}\text { RTC: É a "porcentagem de estudantes que permanecem no } \\
\text { sistema universitário ou completaram o processo de treinamento } \\
\text { satisfatoriamente". }\end{array}$ & - \\
\hline $\begin{array}{l}\text { Pineda-Baez, } \\
\text { Moreno e } \\
\text { Pedraza-Ortiz } \\
\text { (2011) }\end{array}$ & $\begin{array}{l}\text { RTC: afirmam que um programa de retenção estudantil } \\
\text { compreende um conjunto de ações adotadas por uma instituição } \\
\text { para garantir o apoio ao aluno durante sua carreira acadêmica, } \\
\text { de modo que possa concluir seus estudos com êxito }\end{array}$ & Sucesso \\
\hline
\end{tabular}

Fonte: elaborado pelos autores.

A análise do Quadro 1 revela que existem variações na definição de retenção para os autores que diferenciam os conceitos de retenção, para os autores que não diferenciam os conceitos e também para os autores que definem somente a retenção, ressalta-se ainda que não existe um consenso sobre termos relacionados a definição dos autores. $\mathrm{O}$ Centro Nacional de Estatísticas Educacionais do Departamento de Educação dos Estados Unidos, afirma que a diferença entre persistência e retenção é na forma de transferência: enquanto a retenção representa a perspectiva institucional, isto é, a capacidade da instituição de reter seus 
alunos e impedi-los de ir a outra instituição (NATIONAL CENTER FOR EDUCATION STATISTICS, 2000). A persistência representa a perspectiva do aluno, isto é, o fato de um estudante continuar a se inscrever em outra instituição até completar um programa ou obter o diploma. Deixando claro que, as instituições retêm e os estudantes persistem. Os autores Astin (1975), Berger, Ram'rez e Lyon (2005) e Seidman (2005) reforçam a relação de retenção com a capacidade da instituição em reter o estudante, pois estão alinhados a esta afirmação. Observa-se que ao longo de mais de três décadas os autores continuam na mesma direção, mesmo com termos relacionados a sua definição diferentes. Por fim, considerando as definições analisadas e as ponderações apresentadas ao longo desta seção, decidiu-se a adoção, neste estudo a seguinte definição: "Retenção é a capacidade institucional para manter e apoiar os estudantes da admissão até alcançar a graduação com sucesso".

Em relação a esta definição, deve-se observar que a avaliação da retenção do estudante ocorre ao longo de um período e que é necessário um apoio para conclusão da graduação.

\section{PRINCIPAIS MODELOS TEÓRICOS DE RETENÇÃO DE ESTUDANTES, ABORDAGEM E PERSPECTIVAS}

Parte dos modelos teóricos construídos nas últimas cinco décadas foram desenvolvidos para analisar o fenômeno do abandono, no entanto sua estreita relação com a retenção de estudante pavimentou a aplicação dos mesmos para estudos de retenção, mediante observações das características e restrições de cada teoria. A abordagem dos modelos de retenção de estudantes no ensino superior tem sido analisada pelos pesquisadores com base em seis perspectivas teóricas: a psicológica, a sociológica, a econômica, a organizacional, a interacional e a complementar ou integrativa, que compreende as perspectivas (sociológicos, econômicos, organizacionais e psicológicos). A perspectiva psicológica se concentra nos atributos da personalidade do indivíduo. Em contrapartida, a perspectiva sociológica, não se concentra no individual, mas sim nas forças sociais que são externas a instituição educacional como status social, raça e prestígio. A perspectiva econômica se concentra no financiamento financeiro individual que afeta a retenção de estudantes. A perspectiva organizacional está em causa com o impacto de fatores organizacionais, como estrutura burocrática, tamanho e proporção de estudantes. A perspectiva interacional se concentra na influência da interação de indivíduos e fatores ambientais na retenção de estudantes. A perspectiva complementar ou integrativa 
compreende múltiplas perspectivas (sociológicos, econômicos, organizacionais e psicológicos). Considerando a breve apresentação dos modelos teóricos de retenção de estudantes na seção anterior, essa seção, em forma de resumo, e para a melhor visualização dos resultados alcançados, apresenta-se no Quadro 2, a perspectiva, autor, modelo e razão logica como relatado na literatura.

\section{Quadro 2 - Perspectiva, autor, modelo e razão lógica}

\begin{tabular}{|c|c|c|c|}
\hline & & Perspectiv & Psicológica \\
\hline & Autor & Modelo & Razão lógica dos modelos teóricos \\
\hline & $\begin{array}{l}\text { Fishbein e } \\
\text { Ajzen (1975) }\end{array}$ & $\begin{array}{l}\text { Modelo teórico da ação } \\
\text { fundamenta }\end{array}$ & $\begin{array}{l}\text { O comportamento é uma função de intenções } \\
\text { comportamentais que, por sua vez, são uma } \\
\text { função de atitudes e normas subjetivas }\end{array}$ \\
\hline & $\begin{array}{l}\text { Pascarella } \\
(1980)\end{array}$ & $\begin{array}{l}\text { Modelo conceitual de pesquisa } \\
\text { sobre contato informal entre } \\
\text { estudantes e professores }\end{array}$ & $\begin{array}{l}\text { Que não há muita evidência de estudos prévios } \\
\text { para apoiar a influência direta do contato } \\
\text { informal entre estudantes e professores sobre a } \\
\text { persistência dos estudantes. }\end{array}$ \\
\hline & $\begin{array}{l}\text { Bean e } \\
\text { Metzner } \\
(1985)\end{array}$ & $\begin{array}{l}\text { Modelo de desgaste do } \\
\text { Estudante Não Tradicional }\end{array}$ & $\begin{array}{l}\text { Que as decisões dos alunos para abortar ou } \\
\text { continuar sua educação são influenciadas por } \\
\text { suas percepções sobre a utilidade, a satisfação, o } \\
\text { compromisso de objetivo) e níveis de estresse } \\
\text { nas atividades da instituição. }\end{array}$ \\
\hline & $\begin{array}{l}\text { Hurtado e } \\
\text { Carter (1997) }\end{array}$ & Modelo do Sentido de Pertença & $\begin{array}{l}\text { As próprias percepções dos alunos sobre o grau } \\
\text { em que suas experiências são significativas e } \\
\text { contribuem para o seu senso de pertença são } \\
\text { raramente medidas. }\end{array}$ \\
\hline & $\begin{array}{l}\text { Astin (1984, } \\
\text { 1993, p. 21) }\end{array}$ & $\begin{array}{l}\text { (Modelo entrada-ambiente- } \\
\text { resultado (I-E-O)) }\end{array}$ & $\begin{array}{l}\text { Os alunos estão principalmente interessados nos } \\
\text { "benefícios existenciais" da experiência da } \\
\text { faculdade, o que significa, entre outras coisas, a } \\
\text { "satisfação subjetiva associada a ... } \\
\text { envolvimento extracurricular e acadêmico, } \\
\text { atividades recreativas...". }\end{array}$ \\
\hline 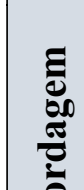 & Padilla (1999) & $\begin{array}{l}\text { Modelo de Expertise dos } \\
\text { Estudantes bem-sucedidos }\end{array}$ & $\begin{array}{l}\text { A capacidade de cada aluno para superar um } \\
\text { conjunto específico de barreiras é a chave para o } \\
\text { sucesso de um aluno. }\end{array}$ \\
\hline$\sum_{4}^{0}$ & Bean e Eaton & Modelo psicológico de retenção & Que este modelo indica que os estudantes são \\
\hline
\end{tabular}




\begin{tabular}{|c|c|c|}
\hline$(2000)$ & do estudante universitário & $\begin{array}{l}\text { seres psicológicos e que as questões coletivas da } \\
\text { sociologia desempenham um papel secundário. } \\
\text { O ambiente social é importante apenas como é } \\
\text { percebido pelo indivíduo. }\end{array}$ \\
\hline \multicolumn{3}{|c|}{ Perspectiva Sociológica } \\
\hline Autor & Modelo & Razão lógica dos modelos teóricos \\
\hline $\begin{array}{l}\text { Spady }(1970, \\
1971)\end{array}$ & $\begin{array}{l}\text { Modelo sociológico de } \\
\text { permanência }\end{array}$ & $\begin{array}{l}\text { Falta de integração dos alunos no ambiente de } \\
\text { ensino superior afeta diretamente a retenção de } \\
\text { estudantes na faculdade }\end{array}$ \\
\hline $\begin{array}{l}\text { Cabrera et al. } \\
\text { (1992) }\end{array}$ & $\begin{array}{l}\text { Modelo integrado de } \\
\text { permanência }\end{array}$ & $\begin{array}{l}\text { Que a ajuda financeira e as atitudes } \\
\text { imediatamente decorrentes se refletem } \\
\text { positivamente, não só para equilibrar as } \\
\text { oportunidades de ingresso de estudantes com } \\
\text { situação socioeconômica inferior, mas também } \\
\text { por facilitarem a integração deste contingente de } \\
\text { estudantes nos componentes acadêmico e social } \\
\text { da instituição. }\end{array}$ \\
\hline $\begin{array}{l}\text { Nora, Barlow } \\
\text { e Crisp (2005) }\end{array}$ & $\begin{array}{l}\text { Modelo do Comprometimento } \\
\text { Estudante-Instituição depois do } \\
\text { primeiro ano }\end{array}$ & $\begin{array}{l}\text { As instituições precisam desenvolver seus } \\
\text { próprios instrumentos para perceber com clareza } \\
\text { as interações entre seus estudantes e a } \\
\text { instituição, as interações dos estudantes com } \\
\text { outros estudantes e professores, as finanças dos } \\
\text { estudantes e o engajamento do estudante com os } \\
\text { sistemas de suporte do campus. }\end{array}$ \\
\hline Swail (2004) & $\begin{array}{l}\text { Modelo geométrico da } \\
\text { perseverança estudantil }\end{array}$ & $\begin{array}{l}\text { A capacidade de ajudar as instituições a } \\
\text { trabalhar proativamente para apoiar a } \\
\text { persistência e a realização dos alunos. }\end{array}$ \\
\hline $\begin{array}{l}\text { Seidman } \\
(2005)\end{array}$ & Modelo de Sucesso do Estudante & $\begin{array}{l}\text { A “identificação precoce” é a identificação, o } \\
\text { mais cedo possível, de um aluno, potencialmente } \\
\text { em risco de não ter sucesso acadêmico ou } \\
\text { pessoal na faculdade. }\end{array}$ \\
\hline \multicolumn{3}{|c|}{ Perspectiva Econômica } \\
\hline Autor & Modelo & Razão lógica dos modelos teóricos \\
\hline $\begin{array}{l}\text { Cabrera, } \\
\text { Stampen e }\end{array}$ & $\begin{array}{l}\text { Modelo de capacidade de } \\
\text { pagamento }\end{array}$ & $\begin{array}{l}\text { Avançaram um modelo em que a capacidade de } \\
\text { pagamento presume interagir tanto com }\end{array}$ \\
\hline
\end{tabular}




\begin{tabular}{|c|c|c|}
\hline Hansen (1990) & & $\begin{array}{l}\text { instituições e variáveis dos indivíduos na } \\
\text { definição de decisões de retirada. }\end{array}$ \\
\hline $\begin{array}{l}\text { St. John, } \\
\text { Paulsen e } \\
\text { Starkey } \\
(1996)\end{array}$ & $\begin{array}{l}\text { Modelo Nexus escolha da } \\
\text { faculdade - persistência }\end{array}$ & $\begin{array}{l}\text { Mudanças na composição do pacote original de } \\
\text { ajuda aluno ou aumentos inesperados nas } \\
\text { propinas e taxas são consideradas como levando } \\
\text { a insatisfação pelo efeito que essas mudanças } \\
\text { têm em os cálculos de custo / benefício originais }\end{array}$ \\
\hline \multicolumn{3}{|c|}{ Perspectiva organizacional } \\
\hline Autor & Modelo & Razão lógica dos modelos teóricos \\
\hline $\begin{array}{l}\text { Brown e } \\
\text { Kayser (1982) }\end{array}$ & Modelo de ajuste educacional & $\begin{array}{l}\text { O ajuste educacional é o grau de } \\
\text { correspondência (satisfação) entre a satisfação } \\
\text { percebida pelos alunos e a satisfação real } \\
\text { (desempenho) em seus programas de } \\
\text { treinamento. }\end{array}$ \\
\hline $\begin{array}{l}\text { Billson e } \\
\text { Terry (1987) }\end{array}$ & Modelo do apoio institucional & $\begin{array}{l}\text { Na premissa de que o apoio institucional através } \\
\text { da infraestrutura organizacional da IESs e o } \\
\text { envolvimento dos alunos reduzirão o atrito, logo } \\
\text { a evasão. }\end{array}$ \\
\hline \multicolumn{3}{|c|}{ Perspectiva Interacional } \\
\hline Autor & Modelo & Razão lógica dos modelos teóricos \\
\hline Tinto (1975) & $\begin{array}{l}\text { Modelo de Integração do } \\
\text { Estudante }\end{array}$ & $\begin{array}{l}\text { A integração dos estudantes na vida acadêmica } \\
\text { e social da instituição precisava ser equilibrada } \\
\text { para um estudante efetivamente persistem na } \\
\text { faculdade. }\end{array}$ \\
\hline Bean (1980) & Modelo de desgaste do estudante & $\begin{array}{l}\text { O abandono da universidade é análogo à } \\
\text { produtividade e que as intenções } \\
\text { comportamentais (permanecer ou abandonar) } \\
\text { são importantes preditores de persistência. }\end{array}$ \\
\hline Tinto (1993) & $\begin{array}{l}\text { Modelo longitudinal do } \\
\text { abandono institucional }\end{array}$ & $\begin{array}{l}\text { A experiência do aluno na faculdade (integração } \\
\text { acadêmica e social) irá modificar continuamente } \\
\text { (enfraquecer ou fortalecer) seu nível de metas e } \\
\text { compromissos iniciais. }\end{array}$ \\
\hline $\begin{array}{l}\text { MacKinnon- } \\
\text { Slaney (1994) }\end{array}$ & $\begin{array}{l}\text { Modelo de desgaste de } \\
\text { estudantes adultos }\end{array}$ & $\begin{array}{l}\text { Que a persistência de adultos para o sucesso no } \\
\text { ensino superior é uma resposta complicada a } \\
\text { uma série de problemas. }\end{array}$ \\
\hline
\end{tabular}




\begin{tabular}{|c|c|c|}
\hline Tinto (1997) & $\begin{array}{l}\text { Modelo de salas de aula, } \\
\text { aprendizagem e permanência }\end{array}$ & $\begin{array}{l}\text { O arranjo de atividades sócio acadêmicas } \\
\text { colaborativas gera um círculo virtuoso, pois a } \\
\text { satisfação nas interações leva a um aumento na } \\
\text { qualidade do esforço feito, pela alocação de } \\
\text { tempo e energia, e leva a melhores resultados } \\
\text { nas esferas social e acadêmica simultaneamente, } \\
\text { o que reforça o sentimento de satisfação. }\end{array}$ \\
\hline \multicolumn{3}{|c|}{ Perspectiva (Complementar ou Integrativa) } \\
\hline Autor & Modelo & Razão lógica dos modelos teóricos \\
\hline Bean (1990) & $\begin{array}{l}\text { Modelo de desgaste longitudinal } \\
\text { do estudante }\end{array}$ & $\begin{array}{l}\text { Que existe uma forte relação entre as intenções } \\
\text { de atitude e comportamento, e que } \\
\text { comportamentos e atitudes refletem intenções. }\end{array}$ \\
\hline $\begin{array}{l}\text { Braxton, } \\
\text { Hirschy e } \\
\text { McClendon } \\
(2004)\end{array}$ & $\begin{array}{l}\text { Modelo Conceitual do abandono } \\
\text { do estudante em IES de Tempo } \\
\text { Parcial }\end{array}$ & $\begin{array}{l}\text { descreve a participação nas comunidades } \\
\text { acadêmicas como uma construção central para } \\
\text { explicar os mecanismos que ligam a experiência } \\
\text { acadêmica para a persistência dos alunos na } \\
\text { faculdade. }\end{array}$ \\
\hline
\end{tabular}

Fonte: elaborado pelos autores.

Conforme se observa nos dados do Quadro 2, a maioria dos autores de modelos teóricos são reconhecidos por suas contribuições pontuais, ou seja, sob uma dessas perspectivas, no entanto, outros autores analisam o tema da retenção de estudantes integrando diferentes perspectivas. A razão lógica neste contexto enfatiza a afirmação dos autores e contribui com os pontos a serem analisados. Os autores Fishbein e Ajzen (1975), Pascarella (1980), Bean e Metzner (1985), Astin (1984, 1993), Padilla (1999) e Bean e Eaton (2000) abordam pontos comuns como o comportamento, satisfação e/ou sucesso em suas análises, estes refletidos pela razão lógica de cada autor em seus modelos teóricos. Dessa forma, percebe-se que há uma relação entre a satisfação dos alunos com a faculdade e a retenção. Esta relação fica enfatizada por Bean e Metzner (1985), que concentraram seus estudos nas percepções dos alunos e na instituição em termos da utilidade, a satisfação e o compromisso do objetivo. Por outro lado, o sucesso é enfatizado no modelo de expertise dos estudantes bem-sucedidos de Padilla (1999), que concluiu que cada aluno possui um grau de especialização e, nesse nível de experiência, subsidia o fracasso ou o sucesso de um estudante. O modelo do sentido de pertença de Hurtado e Carter (1997), concentra sua atenção na percepção dos alunos, estes entendem que o grau de suas experiências é 
significante e contribui para o seu senso de pertença. Nota-se então que apesar dos autores centrarem seus diferentes pontos de atenção para explicar a permanência dos alunos nas instituições, estes nos levam a concluir que existe uma convergência da perspectiva psicológica entre os mesmos.

O estudo de Spady $(1970,1971)$ tem sua atenção direcionado para a integração dos alunos no ambiente de ensino superior, já Cabrera et al. (1992) em seus estudos percebem na ajuda financeira e nas atitudes reflexos positivos, o que facilita a integração do contingente de estudantes nos componentes acadêmico e social da instituição. De acordo com Nora, Barlow e Crisp (2005) as experiências específicas de cada instituição desempenham um papel importante na permanência do estudante à medida que o tempo passa, logo, uma melhor compreensão da natureza destas experiências e de como a instituição pode influenciá-las deve ser construída não a partir de arquivos de dados que combinem dados de instituições de vários tipos, mas de estudos específicos e individuais desta determinada instituição. Swail (2004), em seu modelo geométrico da perseverança estudantil, permite discutir a dinâmica entre fatores cognitivos, sociais e institucionais, todos os quais ocorrem dentro do aluno. $\mathrm{O}$ modelo de sucesso do estudante de Seidman (2005), têm os seus esforços centrados na identificação precoce do estudante potencialmente em risco de não ter sucesso acadêmico ou pessoal na faculdade. $\mathrm{O}$ autor assume que uma intervenção precoce e intensiva pode prover uma intervenção forte o suficiente para efetuar mudanças. A intervenção proporcionará ao aluno uma experiência poderosa o suficiente para que seja eficaz o suficiente para produzir a mudança desejada de comportamento - quanto antes, melhor. Observa-se que os modelos de Spady, (1970, 1971), de Cabrera et al. (1992), de Nora, Barlow e Crisp (2005) e de Seidman (2005) referem-se a assuntos que refletem as forças sociais, tais como satisfação, sucesso, experiências institucionais e experiências com o aluno, apesar dos diferentes assuntos, existe uma convergência para abordagem com perspectiva sociológica.

O modelo de capacidade de pagamento de Cabrera, Stampen e Hansen (1990) concentra-se no aumento da retenção de estudantes e a sua principal fonte de mudança está nos indivíduos que exercem um papel na vida do aluno. Já o modelo nexus escolha da faculdade - persistência de St. John, Paulsen e Starkey (1996) tem os seus esforços concentrados nas mudanças que alteram o pacote original de ajuda financeira do aluno (aumentos inesperados nos valores a serem pagos e nas taxas), o que gera insatisfação pelo efeito que essas mudanças têm nos cálculos de custo/benefício originais. Em outras palavras, a mudança inesperada dentro de uma estimativa de custo/benefício tem implicações na retenção dos estudantes. Nesse sentido os modelos de Cabrera, Stampen e Hansen (1990) e 
St. John, Paulsen e Starkey (1996), convergem para uma abordagem com perspectiva econômica. Brown e Kayser (1982) e Billson e Terry (1987) contribuem com seus modelos através da atenção dirigida a satisfação dos alunos com os serviços prestados pela instituição, estes convergem para uma abordagem com perspectiva organizacional.

Os três estudos de Tinto $(1975,1993,1997)$, vem ao longo dos anos acrescentando contribuições importante para o estudo do abandono e retenção; no seu primeiro modelo, ele descreve que a integração dos estudantes na vida acadêmica e social da instituição precisava ser equilibrada para um estudante efetivamente persistem na faculdade, já no segundo modelo, ele descreve que a experiência do aluno na faculdade (integração acadêmica e social) irá modificar continuamente (enfraquecer ou fortalecer) seu nível de metas e compromissos iniciais e, no seu último modelo, ele descreve que o arranjo de atividades sócio acadêmicas colaborativas gera um círculo virtuoso, pois a satisfação nas interações leva a um aumento na qualidade do esforço feito, pela alocação de tempo e energia, e leva a melhores resultados nas esferas social e acadêmica simultaneamente, o que reforça o sentimento de satisfação. Nesse contexto, observa-se que Tinto (1975) parte de uma modelo base e vem introduzindo melhorias nos modelos seguintes, em busca do melhor entendimento e pavimentação da solução para os fenômenos abandono e retenção. O modelo de desgaste do estudante Bean (1980) enfatiza que as intenções comportamentais são moldadas em um processo em que as crenças formam atitudes e estas, por sua vez, intervêm nas intenções comportamentais, que implicam nas decisões de permanecer ou abandonar a instituição. O estudo de MacKinnonSlaney (1994) tem seus esforços direcionados a três componentes ligados a questões (pessoal, ambiente e aprendizagem) que influenciam a permanência do estudante na instituição, mediante resultado do nível de satisfação e de gratificação do estudante. De maneira geral, os modelos de Tinto (1975, 1993, 1997), Bean (1980) e MacKinnon-Slaney (1994) refletem as preocupações com a satisfação do estudante, o que nos leva ao entendimento de que a abordagem dos modelos converge para uma perspectiva interacionista.

Os autores Bean (1990) e Braxton, Hirschy e McClendon (2004) são os primeiros estudiosos a abordarem o tema retenção de estudantes de múltiplas perspectivas. No modelo de desgaste longitudinal do estudante, Bean (1990) descreve que existe uma forte relação entre as intenções de atitude e comportamento, e que comportamentos e atitudes refletem intenções e que as características de fundo contribuem para a integração acadêmica e social, este modelo inova e aparece como marco na abordagem com perspectivas Sociológica, Econômica, Organizacional e Psicológica (SEOP). Mais de uma década depois o modelo conceitual do abandono do estudante em IES de tempo parcial de Braxton, Hirschy e 
McClendon (2004) aborda, também, o assunto retenção a partir das perspectivas SEOP e descreve a participação nas comunidades acadêmicas como uma construção central para explicar os mecanismos que ligam a experiência acadêmica para a persistência dos alunos na faculdade. Este posicionamento de Braxton, Hirschy e McClendon (2004) é evidenciado nas pesquisas de envolvimento dos alunos (ASTIN, 1984; TINTO, 1997; KUH et al., 2005).

Observa-se que a abordagem com perspectivas SEOP é inovadora e, como toda inovação, têm seu preço, pois a abrangência de diferentes perspectivas leva para um sentido de perda do foco dos problemas relacionados retenção de estudantes, em função de sua ampla abordagem, por outro lado isso é compensado pelas visões que os resultados destes estudos proporcionam para realização de novas pesquisas. Neste contexto os autores Bean (1990) e Braxton, Hirschy e McClendon (2004) nos levam ao entendimento de que estes estudos convergem para uma perspectiva integrativa ou SEOP.

\section{TIPOS DE RETENÇÃO}

Algumas discussões pressupõem a existência de uma variedade de retenção de estudantes que permanecem na instituição ou não. Em função desta variedade, deve-se ter uma atenção em especial na distinção das mesmas, visto que cada uma delas está associado a diferentes fatores e causas. Hagedorn (2006) afirma que a retenção é permanecer na faculdade ou na universidade até obter um diploma, e, identifica quatro tipos de retenção: Retenção institucional, retenção do sistema, retenção dentro de uma especialidade ou disciplina e retenção dentro de um curso.

\section{FATORES CHAVES RELACIONADOS À RETENÇÃO DE ESTUDANTES}

Ao longo dos anos, várias pesquisas sobre retenção de estudantes no ensino superior identificaram fatores que influenciam a retenção na graduação. Considerando os modelos apresentados na seção anterior, esta seção do documento irá resumir os fatores mais citados na literatura, incluindo a preparação acadêmica, integração social, integração acadêmica, compromisso com a instituição, compromisso com o objetivo, ambiente e características demográficas. Os fatores encontrados nos diversos modelos de retenção influenciam, direta ou indiretamente, a permanência dos alunos nos programas até atingir seus objetivos, são apresentados na sequência.

(a) Preparação acadêmica

REAd | Porto Alegre - Vol. 24 - No 3 - Setembro / Dezembro 2018 - p. 155-182 
Bean (1982), Após sua segunda revisão dos modelos de atrito de estudantes de Tinto (1975) e Spady $(1970,1971)$ incorporou idéias de outros estudos teóricos, tais como a importância das intenções na influência do comportamento (FISHBEIN; AJZEN, 1975), o modelo de contato informal da faculdade de estudantes de Pascarella (1980) e seu modelo de atrito (BEAN, 1980). Para fazer isso, Bean (1980) categorizou as variáveis do estudante revisado modelos de atrito em quatro categorias principais: Caracteristicas de fundo, organização, meio ambiente e variáveis atitudinais e de resultados. Bean, enfatiza a importância das características de fundo, incluindo a preparação acadêmica antes da faculdade ou universidade. A qualidade da instrução anterior de um aluno e sua preparação para o trabalho no colégio podem influenciar significativamente se um aluno terá ou não sucesso numa instituição de ensino superior.

(b) Integração social

Os alunos que não se envolvem em atividades no campus da universidade, estão menos propensos à conclusão da graduação. Alguns estudiosos têm enfatizado a importância de uma integração social bem-sucedida para o sucesso dos alunos, está influenciada pela habilidade dos mesmos para desenvolver relacionamentos com colegas e outros estudantes fora dos ambientes acadêmicos rotineiros.

(c) Integração acadêmica

A integração social e a integração acadêmica se influenciam mutuamente. Alguns fatores influenciam a habilidade do estudante de participar ativamente do ambiente acadêmico na instituição (por exemplo: relação com docentes dentro e fora da sala de aula, participação em grupos de estudo, horas dedicadas aos estudos, créditos no semestre, etc.) (CISLAGHI, 2008). Para Pascarella (1985) a integração acadêmica é mais forte do que os compromissos ou objetivos institucionais; portanto, esses compromissos têm um efeito indireto sobre a permanência através da integração social. Pascarella (1985) argumenta que o desenvolvimento e a mudança dos alunos é uma função de cinco conjuntos de variáveis, dos quais estão relacionados: a) Fundo e às características pessoais, b) características estruturais e organizacionais da instituição, c) ambiente ou ambiente institucional, d) interações com estudantes e agentes de socialização e, e) qualidade do esforço dos alunos. Tinto (1975) mede a integração acadêmica bem-sucedida por desempenho de grau e avalia a integração social pelo desenvolvimento e frequência de interação positiva com colegas e professores e envolvimento na atividade extracurricular. Quanto mais fortes forem esses compromissos com a instituição e o objetivo de completar, além de maiores níveis de integração acadêmica e social, menor será o risco de retirada do estudante. 
(d) Compromisso com a instituição

Spady (1971) defende a hipótese de que a decisão de se retirar da faculdade é diretamente influenciada pelo compromisso dos indivíduos com as instituições, tipicamente indicadas pelo desempenho da série. O compromisso institucional é influenciado pela percepção que o estudante tem da instituição que está estudando, sendo este aumentado pelo sentimento de satisfação dos alunos na participação no ambiente educacional e no nível de integração social que eles experimentam. Os aspectos próprios do estudante como indivíduo com suas aspirações, interesses, habilidades e atitudes, refletidas pelas variáveis Compromisso com a instituição (TINTO, 1975, 1993, 1997; PASCARELLA, 1980; CABRERA; NORA; CASTAÑEDA, 1992; MaCKINNON-SLANEY, 1994; NORA; BARLOW; CRISP, 2005) diz respeito à IES escolhida para obter a formação pretendida, como uma instância escolhida entre várias opções ou mesmo como única alternativa existente (CISLAGHI, 2008).

(e) Compromisso com o objetivo

Tinto (1975) argumentou que o estudante menos comprometido com o objetivo de ganhar um diploma universitário, tem maior probabilidade de abandonar a faculdade. Pesquisas em estudantes universitários hispânicos mostraram que seus compromissos com o objetivo de obter um diploma e a medida em que eles se envolvem em discussões acadêmicas e atividades dentro e fora do campus (CABRERA; NORA; CASTAÑEDA, 1993), bem como o seu primeiro-ano de desempenho acadêmico (NORA; CABRERA, 1996), influencia suas decisões em permanecer na faculdade. O compromisso com o objetivo é influenciado pela percepção que o estudante tem da qualidade do curso, decorrente da formação que recebe, e utilidade do diploma correspondente frente ao esforço necessário para manter seu vínculo (intenção de concluir o curso, intenção de exercer a profissão).

(f) Ambiente

O ambiente inclui fatores como finanças, trabalho e família, estes estão relacionadas diretamente ou indiretamente com o que acontece durante o curso do programa que pode afetar o aluno e, portanto, os resultados medidos. Alguns estudos têm mostrado evidências de que as finanças têm um impacto nas experiências e no sucesso dos estudantes universitários em geral (PASCARELLA; TERENZINI, 1991, 2005).

(g) Características demográficas

Características demográficas, habilidades e atitudes que os estudantes trazem do ensino médio para a faculdade influenciam positivamente sua decisão de permanência na instituição. Os recursos acadêmicos que os alunos trazem para a faculdade, medidos 
principalmente em termos de qualidade e rigor do currículo do ensino médio, emergiram como um dos maiores preditores na graduação do curso.

\section{INDICADORES DE RETENÇÃO}

Altas taxas de abandono dos estudantes no ensino superior tem sido um motivo de preocupação crescente em muitas instituições de ensino superior. $O$ entendimento da complexidade desde fenômeno por estudiosos através de pesquisas e teorias exploram o impacto de vários fatores individuais, sociais e organizacionais sobre a retenção de estudantes no ensino superior (TINTO, 1975, 1993; ASTIN, 1984; PASCARELLA; TERENZINI, 1991). Os conceitos e os indicadores de desempenho nacionais, quando comparados com os internacionais, costumam ser diferente, o que tem gerado dificuldades de generalização. A retenção de estudante por ser um conceito completo, confuso e dependendo do contexto tornar-se desafiador para medir e controlar. O conceito de retenção, no entanto, é muito mais complicado devido à prevalência de inscrição de estudantes em diversas instituições diferentes ao longo de sua carreira educacional. Apesar da variedade de estudos estatísticos, não existe uma fórmula única para medir a retenção de estudantes. Algumas fontes, na literatura, indicam haver seis práticas de medição dominantes, a saber: as taxas de abandono, as taxas de conclusão, as taxas de desgaste, as taxas de graduação, as taxas de sobrevivência e as taxas de retenção. Estas medidas são as mais comumente aceites e amplamente utilizadas. Considerando a definição da taxa de retenção "A percentagem de um grupo definido de estudantes que permanecem na educação por algum tempo definido." As variáveis e indicadores relacionados a essa taxa de retenção são listadas conforme a seguir:

(a) preparação acadêmica - resultados acadêmicos e Atividades acadêmicas enquanto matriculados;

(b) integração social - apoio institucional para integração (processos), percepção do relacionamento com colegas campus e realização de atividades no campus;

(c) integração acadêmica - satisfação com docentes em classe, relacionamento com docentes extraclasse, reforço para ingressantes com base frágil e dedicação às atividades acadêmicas;

(d) compromisso com a instituição - grau de satisfação geral com a IES, conceito relativo do curso na IES, nível de apoio encontrado para a permanência e natureza da instituição (paga x gratuita); 
(e) compromisso com o objetivo (currículo, curso e interesses pessoais) = Adequação do currículo à formação esperada, grau de formação profissionalizante oferecida, dificuldade na obtenção de créditos, adequação da infraestrutura de apoio ao ensino, grau de satisfação geral com o curso, intenção de completar o curso e intenção de exercer a profissão

(f) ambiente (finanças, trabalho e família) - dificuldades financeiras para sustento, tempo dedicado a atividades profissionais fora do campus, necessidade de viajar a trabalho e número de dependentes;

(g) características demográficas - antecedentes educacionais, currículo do ensino médio (bagagem) e padrão de comportamento.

\section{DESENVOLVIMENTOS FUTUROS, CONTRIBUIÇÕES E LIMITAÇÕES DAS TEORIAS DA RETENÇÃO DE ESTUDANTES}

Modelos de retenção foram desenvolvidos basicamente para auxiliar as instituições a identificarem formas de reduzir as taxas de evasão dos estudantes, promover a permanência, melhorar seus programas educacionais, etc. Sugestões ou mesmo recomendações dos estudiosos do assunto tem refletido positivamente nas instituições de ensino. Algumas instituições preocupadas com a questão da evasão vêm buscando entender o porquê das altas taxas da evasão e como melhorar suas intervenções no sentido de apoiar os estudantes a concluir com êxito seus estudos e adequar as suas características organizacionais as demandas do mercado educacional. Muitos dos modelos de retenção são baseados em uma diversidade de teorias, perspectivas e normalmente cercados por questões, objetivos, fatores e variáveis complexas. As várias questões relacionadas ao problema das altas taxas da evasão de estudantes têm feito com que os pesquisadores saiam de uma abordagem com perspectiva pontual para uma abordagem mais abrangente e integrativa, preocupada com soluções mais construtivas. A medida que estas pesquisas avançam, há uma tendência natural de ajuste ou combinação das perspectivas nos modelos. O presente estudo contribui no sentido de lançar luz sobre dois pontos importantes para o estudo dos modelos da retenção de estudantes. O primeiro ponto chama atenção para dois modelos teóricos que possuem uma abordagem integrativa, e, que merecem destaque por compreenderem múltiplas perspectivas (sociológicos, econômicos, organizacionais e psicológicos), são eles: Modelo de desgaste longitudinal do estudante Bean (1990) que explicar o processo da evasão de estudantes e o modelo Conceitual do abandono do estudante em IES de Tempo Parcial de Braxton, Hirschy e McClendon (2004) que explicar os mecanismos que ligam a experiência acadêmica para a

REAd | Porto Alegre - Vol. 24 - No 3 - Setembro / Dezembro 2018 - p. 155-182 
persistência dos alunos na faculdade. Essas múltiplas perspectivas ampliam as dimensões dos modelos e ajudam explicar os processos de retenção, abandono e persistência de estudantes nas instituições de ensino. O segundo ponto diz respeito a cinco modelos teóricos notáveis, que incluem o modelo teórico de desgaste de Bean e Metzner (1985), o modelo integrado de permanência de Cabrera et al. (1992), o modelo longitudinal do abandono institucional de Tinto (1993), modelo geométrico da perseverança estudantil de Swail (2004) e a fórmula de Seidman (2005) para a retenção de estudantes. Esses cinco modelos vêm servindo de base na construção de modelos intervencionistas para apoiar ações institucionais na retenção de estudantes. O modelo de Bean e Metzner (1985) concentra-se em fatores externos a instituição e fornece uma visão geral de socialização dos ambientes universitários no processo de desgaste; o modelo de Cabrera et al. (1992) explicar a retenção de estudantes e examinar o convergente e validade discriminante entre Tinto (1975) e Bean (1980) e à teoria de Bean e Metzner (1985); o modelo de Tinto (1993) centra-se nos fatores críticos da integração social e acadêmica, e compromissos com o objetivo e com a instituição; o modelo de Swail (2004) se concentra em fatores institucionais que têm o impacto mais significativo na retenção de estudantes, em quanto o modelo de Seidman (2005) centra-se na identificação precoce de estudantes em risco e na aplicação de estratégias de intervenção para esses alunos. Baseado nesta pesquisa, até o presente momento, somente os modelos teóricos de Bean e Metzner (1985), Cabrera et al. (1992) e Tinto (1993) foram amplamente testados e revisados, por outro lado os modelos de Swail (2004) e Seidman (2005), não parecem ter sido validados até à presente data. Assim, levanta-se a importância da realização da validação destes e outros modelos, e que seus resultados apontem para um maior aperfeiçoamento dos mesmos e eleve o nível de generalização das descobertas.

Ressaltamos que identificação e compreensão das perspectivas dos modelos teóricos de retenção é de fundamental importância para os educadores, administradores e formuladores de políticas na construção das estratégias, políticas de apoio e intervenções das instituições de ensino.

Modelos teóricos que lidam com questões da retenção de estudantes no ensino superior, têm suas limitações. Uma das limitações mais conhecidas dos estudos de retenção de estudantes diz respeito à sua generalização (JEFFREYS, 2012). A maioria dos estudos de retenção de estudantes é realizada em instituições particulares e seus resultados geralmente não são facilmente generalizados para outras instituições (BOSTON; ICE, 2011). Mesmo, modelos com abordagens e perspectivas iguais se observa que, estas caminham para diferentes soluções, pois tem características e circunstâncias únicas que difícultam a 
generalização de seus achados para casos diferentes. Outras limitações conhecidas nos estudos dos modelos de retenção de estudantes estão relacionadas aos grupos de estudantes tradicionais e não tradicionais, aos tipos de instituições públicas e privadas e às estratégias de pesquisa que são utilizadas. Uma das críticas dos estudos de retenção de estudantes é que eles se concentraram, principalmente, em instituições acadêmicas tradicionais e tipos tradicionais de estudantes (BEAN; METZNER, 1985; BRAXTON; LEE, 2005).

\section{CONSIDERAÇÕES FINAIS}

A pesquisa obteve como resultado vinte três modelos teóricos de retenção com suas respectivas abordagens e perspectivas. Dessas abordagens, sete são modelos com perspectivas psicológicas que em sua maioria tratam das percepções sobre a utilidade, a satisfação, o compromisso de objetivo e níveis de estresse dos estudantes nas atividades da instituição. Cinco são modelos com perspectivas sociológicas que tratam normalmente da interação e integração dos estudantes no ambiente de ensino superior. Duas são modelos com perspectivas econômicas que dizem respeito à capacidade de pagamento do estudante e sua sensibilidade para com as alterações nos pacotes firmados inicialmente. Duas são modelos com perspectivas organizações que dizem respeito a (satisfação) entre a satisfação percebida pelos estudantes e a satisfação real (desempenho) em seus programas de treinamento. Cinco são modelos com perspectivas interacionais que tratam do arranjo de atividades sócioacadêmicas colaborativas e o sentimento de satisfação. Duas são modelos com perspectivas complementar ou integrativas, e tratam das intenções de atitude e comportamento do estudante. Assim, este estudo cumpriu seu objetivo, pois fornece aos educadores, administradores e formuladores de políticas uma visão abrangente dos principais modelos teóricos de retenção de estudantes nas últimas oito décadas, considerando as abordagens e perspectivas para auxiliar nas questões das retiradas do estudante, encontrar padrões e diferenciar termos e suas relações com a retenção. A maioria dos autores de modelos teóricos são reconhecidos por suas contribuições pontuais nas soluções das questões relacionadas a retirada voluntaria do estudante, ou seja, estes utilizam somente uma das perspectivas teóricas em seu modelo. No entanto, Bean (1990) e Braxton, Hirschy e McClendon (2004) deram início a um novo tipo de abordagem para solução das questões relacionadas a retirada voluntaria do estudante. Esses estudiosos partiram para uma abordagem mais abrangentes do tema retenção de estudantes, integrando múltiplas perspectivas teóricas e enriquecendo suas pesquisas. O Modelo de desgaste longitudinal do estudante de Bean (1990), por exemplo, é 
um modelo integrativo que aborda o ponto de partida dos seus estudos de múltiplas perspectivas teóricas e que tem feito contribuições importantes para o estudo do abandono e retenção de estudantes do ponto de vista psicológico e interacionista. Para o autor, existe uma forte relação entre as intenções de atitude e comportamento, e que comportamentos e atitudes refletem intenções. Já o Modelo conceitual do abandono do estudante em IES de tempo parcial de Braxton et al. (2004) tem se tornado um importante avanço teórico na pesquisa de retenção, já que conceitua múltiplos perspectivas, a saber: econômicas, organizacionais, psicológicas e sociológicas forças que influenciam estudantes de tempo parcial na sua persistência na faculdade. Os autores postulam que quanto mais os alunos participam, envolvem e se envolvem em atividades acadêmicas e comunidades de aprendizado, menos provável que eles vão deixar a instituição. Alguns dos modelos teóricos de encontrados neste estudo para soluções das questões relacionadas a retirada voluntaria do estudante não foram testados em diferentes ambientes. Assim, sugere-se que futuras investigações dos modelos de retenção de estudantes, sejam feitas especificamente com base nos modelos de retenção testados e validados.

\section{REFERÊNCIAS}

ARGENTINA. Ministério da Educação, Ciência e Tecnologia da República Argentina. Agencia Interamericana para Cooperação e Desenvolvimento. Documento base do projeto: estratégias e materiais pedagógicos para retenção escolar. Buenos Aires, 2003. Disponível em: http://www.oei.es/quipu/proyecto_retencion_escolar_OEA.pdf. Acesso em: 24 nov. 2017.

ASTIN, Alexander. Preventing students from dropping out. MMichigann: Jossey-Bass, 1975.

ASTIN, Alexander. Student involvement: a developmental theory for higher education.

Journal of College Student Personnel, Washington, DC, v. 25, n. 4, p. 297-308, Jan. 1984. ASTIN, Alexander. What matters in college? Four critical years revisited. San Francisco: Jossey-Bass Publisher, 1993.

BEAN, John. Conceptual models of student attrition: How theory can help the institutional researcher. In: PASCARELLA, Ernest (Ed.). Studying student attrition. San Francisco: Jossey-3ass, 1982. p. 17-33.

BEAN, John. Dropouts and turnover: the synthesis and test of a causal model of student attrition. Research in Higher Education, Switzerland, v. 12, n. 2, p. 155-187, Spr. 1980. 
BEAN, John. Why students leave: Insights from research. In: HOSSLER, Don. (Eds.). The strategic management of college enrollments. San Francisco: Jossey-Bass, 1990. p. 147169.

BEAN, John; EATON, Shevawn. A psychological model of college student retention. In: BRAXTON, John (Ed.). Reworking the departure puzzle: new theory and research on college student retention. Nashville: University of Vanberbilt Press, 2000. p. 48-62. BEAN, John; METZNER, Barbara. A conceptual model of nontraditional undergraduate student attrition. Review of Educational Research, Washington, DC, v. 55, n. 4, p. 485-540, Win. 1985.

BERGER, Joseph; RAM'REZ, Gerardo Blanco; LYONs, Susan. A historical look at retention. In: SEIDMAN, Alan. College student retention: formula for student success. Westport, CT: American Council on Education/Praeger, 2005. p. 7-34.

BERGER, Joseph; RAM'REZ, Gerardo Blanco; LYONS, Susan. Past to present: a historical look at retention. In: SEIDMAN, Alan. (Ed.). College student retention: formula for student success. New York: Rowman \& Littlefield, 2012. p. 7-34.

BILLSON, Janet Mancini; TERRY, Margaret Brooks. A student retention model for higher education. College and University, London, v. 62, n. 4, p. 290-305, 1987. Disponível em: http://www.eric.ed.gov/ERICWebPortal/search/detailmini.jsp?_nfpb=true\&_\&ERICE xtSearch_SearchValue_0=EJ359544\&ERICExtSearch_SearchType_0=no\&accno=E J359544. Acesso em: 6 jan. 2018.

BOSTON, Wallace; ICE, Phill. Assessing retention in online learning: an administrative perspective. Online Journal of Distance Learning Administration, v. 14, n. 2, p. 1-12, 2011.

BRASIL. Ministério da Educação. Secretaria da Educação Superior. Diplomação, retenção e evasão nos cursos de graduação em Instituições de Ensino Superior Públicas: relatório da Comissão Especial de Estudos sobre Evasão nas Universidades Públicas Brasileiras. Brasília, DF: Andifes, 1997.

BRAXTON, John; HIRSCHY, Amy; McCLENDON, Shederick. Understanding and reducing college student departure. Ashe-ERIC Higher Education Report, Washington, DC, v. 30, n. 3, p. 1-103, Apr. 2004.

BRAXTON, John; LEE, S. Toward reliable knowledge about college student departure. In: SEIDMAN, A. (Ed.). College student retention: formula for student success. Westport, CT: American Council on Education/Praeger, 2005. p. 107-127. 
BROWN, James; KAYSER, Terrence. The transition of special needs learners into postsecondary vocational education. 1982. Disponível em:

http://eric.ed.gov/?id=ED217298. Acesso em: 6 jan. 2018.

CABRERA, Alberto et al. The convergence between two theories of college persistence. The Journal of Higher Education, London, v. 63, n. 2, p. 143-164, Mar./Apr. 1992.

CABRERA, Alberto; NORA, Amaury; CASTAÑEDA, Maria. College persistence: structural equations modeling test of and integrated models of student retention. The Journal of Higher Education, London, v. 64, n. 2, p. 123-139, Mar./Apr. 1993.

CABRERA, Alberto; NORA, Amaury; CASTAÑEDA, Maria. The role of finances in the persistence process: a structural model. Research in Higher Education, Switzerland, v. 33, n. 5, p. 571-593, Oct. 1992.

CABRERA, Alberto; STAMPEN, Jacob; HANSEN, W. Lee. Exploring the effects of ability to pay on collage persistence. The Review of Higher Education, Maryland, v. 13, p. 303336, Jan. 1990.

CISLAGHI, Renato. Um modelo de sistema de gestão do conhecimento em um framework para a promoção da permanência discente no ensino de graduação. 2008. 258 f. Tese (Doutorado) - Programa de Pós-Graduação em Engenharia e Gestão do Conhecimento, Universidade Federal de Santa Catarina, Florianópolis, 2008.

DURKHEIM, Émile. Suicide. Glencoe, IL: The Free Press, 1951.

FISHBEIN, Martin; AJZEN, Icek. Belief, attitude, intention and behavior: an introduction to theory and research. Reading, MA: Addison-Wesley, 1975. Disponível em: http://people.umass.edu/aizen/f\&a1975.html. Acesso em: 6 jan. 2018.

GUEVARA, Luz Elba Torres. Estado del arte de la retención de estudiantes de la educación superior. Bogotá: Pontificia Universidad Javeriana, 2010. Disponivel em: http://pujportal.javeriana.edu.co/portal/page/portal/portal_version_2009_2010/resources_v4/estado_de 1 arte de la_retencion_de estudiantes.pdf. Acesso em: 24 nov. 2017.

HABLEY, Wesley; BLOOM, Jennifer; ROBBINS, Steve. Increasing Persistence: researchbased strategies for college student success. New Jersey: John Wiley \& Sons, 2012.

HAGEDORN, Linda Serra. How to define retention: a new look at an old problem. Los Angeles: ERIC, 2006. Disponível em: https://eric.ed.gov/?id=ED493674. Acesso em: 20 ago. 2017.

HIMMEL, Erika. Modelos de análisis de la deserción estudiantil en la educación superior. Revista Calidad en la Educación, Santiago, n. 17, p. 91-108, 2002. Disponível em: 
http://www.cse.cl/public/secciones/seccionpublicaciones/publicaciones_revista_calidad_detal le. aspx?idPublicacion=35 Hollinnngsworth. Acesso em: 24 nov. 2017.

HURTADO, Sylvia; CARTER, Deborah. Effects of college transition and perceptions of the campus racial climate on Latino college students' sense of belonging. Sociology of

Education, Georgia, v. 70, p. 324-345, Oct. 1997.

JEFFREYS, Marianne R. Nursing student retention: understanding the process and making a difference. New York: Springer Publishing Company, 2012.

KERKA, Sandra. Adult learner: retention revisited. Columbus: ERIC, 1995. (ERIC Digest, 166). Disponível em: https://eric.ed.gov/?id=ED389880. Acesso em: 24 nov. 2017. KUH, George et al. Assessing conditions to enhance educational effectiveness: the inventory for Student Engagement and Success. San Francisco: Jossey-Bass, 2005.

LENNING, Oscar; BEAL, Philip; SAUER, Ken. Retention and attrition: evidence for action and research. Boulder, CO: National Center for Higher Education Management Systems, 1980.

LEVITZ, Randi. What's working right now in student retention! building blocks for retention success: the basis for recruiting success. In: NATIONAL CONFERENCE FOR STUDENT RETENTION, 2001, New Orleans. Paper... New Orleans, 2001.

MACKINNON-SLANEY, Fiona. The adult persistence in learning model. Journal of Counseling \& Development, Virginia, v. 72, n. 3, p. 268-275, Jan. 1994.

NATIONAL CENTER FOR EDUCATION STATISTICS. The condition of education2000. New York, 2000. Disponível em: https://nces.ed.gov/pubs2000/2000062.pdf. Acesso em: 24 nov. 2017.

NATIONAL CENTER FOR EDUCATION STATISTICS. The condition of education. 2012. Disponível em: http://nces.ed.gov/programs/coe. Acesso em: 24 nov. 2017. NORA, Amaury; BARLOW, Elizabeth; CRISP, Gloria. Student persistence and degree attainment beyond the first year in college. In: SEIDMAN, Alan (Eds.). College student retention: formula for student success. Westport, CT: American Council on Education/Praeger, 2005. p. 229-250.

NORA, Amaury; CABRERA, Alberto. The role of perceptions of prejudice and discrimination on the adjustment of minority students to college. The Journal of Higher Education, London, v. 67, n. 2, p. 120-148, Mar./Apr. 1996.

PADILLA, Raymond. College student retention: focus on success. Journal of College Student Retention: Research, Theory \& Practice, New York, v. 1, p. 131-145, Aug. 1999. 
PASCARELLA, Ernest. Student-faculty informal contact and college outcomes. Review of Educational Research, Washington, DC, v. 50, v. 4, p. 545-59, Dec. 1980.

PASCARELLA, Ernest; TERENZINI, Patrick. How college affects students: findings and insights from twenty years. Oxford: Jossey-Bass Publishers, 1991.

PASCARELLA, Ernest; TERENZINI, Patrick. How college affects students: a third decade of research. San Francisco: John Wiley \& Sons, 2005. v. 2.

PASCARELLA, Even. College environmental influences on learning and cognitive development: a critical review and synthesis. In: SMART, John. (Ed.). Higher education: handbook of theory and research. New York: Agathon, 1985. p. 1-61.

PINEDA-BAEZ, Clelia; PEDRAZA-ORTIZ, Alexandra; MORENO, Iván Darío. Efectividad de las estrategias de retención universitaria: la función del docente. Educación y Educadores, Bogotá, v. 14, n. 1, p. 119-135, mar. 2011.

PRICE, James. The study of turnover. Ames: Iowa State University Press, 1977. SANTOS, Adilson Pereira dos. Diagnóstico do fluxo de estudantes nos cursos de graduação da UFOP: retenção, diplomação e evasão. Revista da Rede de Avaliação da Educação Superior, Sorocaba, v. 4, n. 4, p. 55-68, dez. 1999.

SEIDMAN, Alan. College student retention: formula for student success. Westport, CT: American Councilon Education/Praeger, 2005.

SPADY, William. Dropouts from higher education: an interdisciplinary review and synthesis. Interchange, Netherlands, v. 1, n. 1, p. 64-85, Apr. 1970.

SPADY, William. Dropouts from higher education: toward an empirical model. Interchange, v. 2, n. 3, p. 38-62, Sep. 1971.

St. JOHN, Edward; PAULSEN, Michael; STARKEY, Johnny. The nexus between college choice and persistence. Research in Higher Education, Switzerland, v. 37, n. 2, p. 175-220, Apr. 1996.

SUMMERSKILL, John. Dropouts from college. In: SANFORD, Nevitt. (Ed.). The American College. New York: John Wiley and Sons, 1962. p. 627-657.

SWAIL, Watson Scott. The development of a conceptual framework to increase student retention in Science, Engineering and Mathematics programs at minority institutions of Higher Education. 1995. Tesis (Doctoral) - George Washington University, Washington, 1995. Disponível em:

http://www.eric.ed.gov/ERICWebPortal/contentdelivery/servlet/ERICServlet?accno=ED3969 21. Acesso em: 28 nov. 2017. 
SWAIL, Watson Scott. Value added: the costs and benefits of college preparatory programs. 2004. Disponível em: http://educationalpolicy.org/pdf/value added.pdf. Acesso em: 6 nov. 2017.

TINTO, Vicent. Classrooms as communities: exploring the educational character of student persistence. The Journal of Higher Education, London, v. 68, n. 6, p. 599-624, Nov./Dec. 1997.

TINTO, Vicent. Dropout from higher education: a theoretical synthesis of recent research.

Review of Educational Research, Washington, DC, v. 45, n. 1, p. 89-125, Mar. 1975.

TINTO, Vicent. Leaving college: rethinking the causes and cures of student attrition. $2^{\text {nd }}$ ed. Chicago: University of Chicago Press, 1993.

UNIVERSIDAD NACIONAL DE COLOMBIA. Estudio de la deserción estudiantil en la educación superior en Colombia: estado del arte sobre la retención estudiantil. Bogotá, 2002. Convenio 107/2002 UN ICFES.

UNIVERSIDAD TECNOLÓGICA DE PEREIRA. Manual del manejo de la información: oficina de planeación, administración de la información estratégica: indicador adaptación e integración a la vida universitaria. 2008. Disponible en: $<$ http://planea.utp.edu.co/PDI_20072019/Protocolos/BIE1201.pdf>. Acesso en: 10 nov. 2017.

VAN GENNEP, Arnold. The rites of passage. Chicago: Phoenix Books/University of Chicago Press, 1960. 